\section{QUANTUM OPTICS}

\section{Silicon integration}

Nano Lett. http://doi.org/gckz6q (2017)

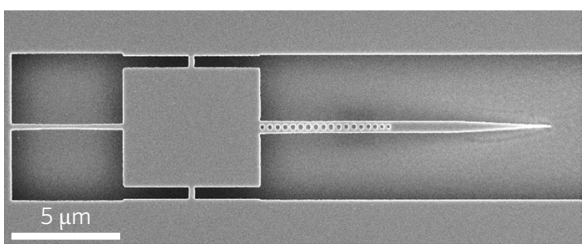

Credit: American Chemical Society it placed onto the silicon waveguide. With the use of a y-shaped Si waveguide, the single-photon nature of the InAs QDs emitting around 1,300 nm was confirmed by a Hanbury-Brown and Twiss measurement.

https://doi.org/10.1038/s41566-017-0082-3

\section{SOLAR ENERGY}

\section{Dynamic absorbers \\ Nat. Commun. 8, 1478 (2017)}

The development of chip-based quantum photonic devices requires the successful integration of single-photon sources with waveguide circuitry. Now, Je-Hyung Kim and co-workers from the USA and the Republic of Korea have developed a pick-and-place technique to transfer a quantum emitter composed of an InP nanobeam containing InAs quantum dots (QDs) directly onto a $\mathrm{Si}$ waveguide with nanometre-scale precision. The InP nanobeam (500 nm wide and $280 \mathrm{~nm}$ thick) and Si waveguide (400 $\mathrm{nm}$ wide and $220 \mathrm{~nm}$ thick) were both fabricated by using electron-beam lithography. The nanobeam is tapered at an angle of $6^{\circ}$ over a length of $5 \mu \mathrm{m}$ in order to adiabatically convert the photonic mode into the correct shape for the $\mathrm{Si}$ waveguide. The pick-and-place technique relies on the use of a focused ion beam to detach the InP nanobeam from a substrate by cutting tethers. The nanobeam is then picked up by the tip of a microprobe (it adheres to the nanobeam due to the van der Waals force) and a scanning electron microscope is used to image the position of the nanobeam as
The storage of solar energy using the phase change of a material, for example, the melting of a solid into liquid, is often a slow process due to the thermal properties of the material involved. Now, Zhongyong Wang and colleagues have reported a means to speed up the process and increase the amount of stored energy by a factor of approximately three by magnetic control of optical absorber distribution. The authors use $\mathrm{Fe}_{3} \mathrm{O}_{4} @$ graphene nanoparticles in a paraffin host. The graphene component of the nanoparticles yields strong optical absorption of the solar radiation while the $\mathrm{Fe}_{3} \mathrm{O}_{4}$ enables magnetic manipulation. Once the surface of the composite material is melted by photothermal conversion, the nanoparticles in the liquid are attracted to a side wall by a magnet within a few seconds. This removal of the optical absorbers allows better penetration of light deeper into the material allowing greater deposition of solar energy.

\section{CARBON NANOTUBES}

\section{Ultrasmall optical memory \\ ACS Photon. http://doi.org/cg2f (2017)}

NH Nanomaterials that exhibit optical bistability provide routes for high-density integration of optical memory and switches. Now, Takushi Uda and co-workers from RIKEN and the University of Tokyo in Japan present an optical memory based on individual carbon nanotubes where excitonic resonance shifts induced by molecular adsorption and desorption give rise to optical bistability. The device in question is composed of individual carbon nanotubes suspended over trenches on silicon substrates. The nanotubes are excited by a laser and their photoluminescence spectra captured by a microscopy system. The team observed two different emission energy peaks during an up-sweep and down-sweep of laser excitation power, indicating the presence of optical bistability and the existence of a hot desorbed state and a cold adsorbed state. Reversible and reproducible optical memory operation using the two states was confirmed by time-resolved measurements. The findings aid the design of photonic memory devices that are a few orders of magnitude smaller than the optical diffraction limit.

https://doi.org/10.1038/s41566-017-0084-1

\section{IMAGING}

\section{Needle ultrasound}

Light Sci. Appl. 6, e17103 (2017)

Heart surgery and other complex operations could be made easier thanks to the use of optically generated ultrasound imaging that can be performed at the end of a needle. A team of scientists from University College London and Queen Mary University of London in the UK have developed an imaging system whereby two optical fibres are placed inside a hollow needle to locally generate and detect ultrasound. The generation fibre is equipped with a special absorbing coating at the end that on exposure to laser light generates ultrasound due to the photoacoustic effect. Ultrasound reflections from the surrounding tissue are detected using light in the receiving fibre, which is passed into a Fabry-Perot cavity. The approach has been demonstrated with cardiac surgery on a pig. phenomenon. The findings may also be useful for developing an optical instrument for early detection of retinal disease or one that can evaluate the condition of the underlying visual system in the case of someone with nearly opaque eyes.

https://doi.org/10.1038/s41566-017-0081-4 